\title{
Optimal Parameter Matching for Working Condition of Combine Harvester
}

\author{
Min Liao ${ }^{1, a^{*}}$, Qiang Wang ${ }^{1, b}$ and Huapei Zhang ${ }^{1, c}$ \\ ${ }^{1}$ School of Mechanical Engineering, Xihua University, Chengdu, Sichuan 610039, PR China \\ aliaominxhu@163.com, bwq_py123@163.com, '952309072@qq.com
}

\begin{abstract}
Keywords: Parameter matching; Genetic algorithm; Fuzzy logic control; Combine harvester
Abstract. To improve harvesting productivity, optimal parameter matching for working condition of combine harvester is studied. Interrelationship of crucial working parameter of combine harvester has been discussed. And a fuzzy logic controller (FLC) was presented by making use of feeding rate, grain moisture content, forward speed and cylinder speed. Fitness function of genetic algorithm was established based on the highest productivity of combine. Genetic-fuzzy algorithm (GFA) was utilized to solve the optimal combination. The results show that the presented FLC and GFA are feasible and efficiency.
\end{abstract}

\section{Introduction}

Common problems for a combine are low efficiency and harvesting quality, e.g. big grain loss and Grain/Material other than Grain (G/MOG) ratio. Many factors can affect the grain loss and G/MOG ratio because grain harvesting is of considerable complexity, and there is no effective strategy to completely control behaviors of the complex system, obtaining optimal parameter matching for working condition of combine harvester. Since yield and crop properties (e.g. moisture content, grain/straw ratio) can change in one or different field because of different soil fertility and water condition, a combine operator should change continuously working parameters to improve performance. Then it is evident important to obtain the crucial parameter matching of a combine and grain crops, such as feeding rate, forward speed, cylinder speed, grain moisture content and G/MOG ratio, etc.

Intelligent control approaches based a programmable logic controller (PLC) have been extensively studied but the crucial parameter matching and its optimal algorithm have received less attention, even though the key parameter harmonizing influences combine performance. To achieve minimal grain loss and maintain maximum efficiency and throughput, a control system [1] was designed by making use of microcontroller, portable computer and communication board to automatically adjust three main parameters, i.e. federate, sieve airflow and cylinder speed. Similarly, an intelligent control system [2] based on microprocessor technology was developed to detect entrainment loss, cleaning loss and cylinder speed, so that the grain loss was greatly reduced. In addition, a fuzzy logic controller [3] was implemented by a PLC to carry out fuzzy logic algorithm and data processing, such that the key parameters of a combine could be automatically regulated on basis of the measured losses at straw walker and sieve.

Parameter matching is worthy of study from high efficiency and precision harvesting point of view, and it can greatly promote intelligent design of a combine harvester and improve working performance. The objective of present work is to obtain a set of optimal parameters for working condition of combine harvester, resulting in a high behavior of productivity, and fuzzy logic controller is studied incorporating genetic algorithm to address the problem.

\section{Interrelationship of crucial working parameter of combine harvester}

Forward speed versus feeding rate. Relationship between feeding rate and forward speed was established on basis of field experiment of harvesting. And the experimental results revealed that 
forward speed showed an approximately linear increase as feeding rate increased. Interrelationship between feeding rate and forward speed [4] was defined by

$$
v_{m}=100 q / B Q
$$

where $v_{m}, q, B, Q$ are forward speed, feeding rate, cutting width and crop density, respectively. Here cutting width and crop density can be taken as constant parameters.

Feeding rate versus cylinder speed. Generally, correlation between feeding rate and cylinder speed can be determined empirically. As feeding rate increases, cylinder speed is also increased to some extent. If cylinder speed keeps unchanged, forward speed of combine should be automatically adjusted to regulate feeding rate simultaneously.

Cylinder speed versus grain moisture content. Critical cylinder speed is determined by grain damage. Based on contact mechanics analysis between a grain kernel and a threshing component, the critical speed [5] causing plastic deformation was formulated by

$$
v_{s}^{2}=2 \pi n R \approx 106.96 \frac{\sigma_{s}^{5} R_{e}^{3}}{m_{2} E^{* 4} F_{2}}
$$

where $v_{s}$ shows the critical speed, $n$ and $R$ respectively indicate rotary speed and radius of threshing cylinder, $\sigma_{s}$ denotes grain yield stress in uniaxial compression, $R_{e}$ indicates relative radius of curvature when grain kernel and threshing element collide each other, $m_{2}$ and $F_{2}$ respectively show grain mass and correction coefficient, $E^{*}$ is determined by elastic modulus and Poisson ratios of grain kernel and threshing element. Here the elastic modulus and Poisson ratio of threshing element remain unchanged. The cylinder speed should be increased as the grain elastic modulus decreases, i.e. a lower grain elastic modulus brings about a higher cylinder speed.

Grain moisture content directly affects grain mechanical properties. The experimental analysis found that higher grain moisture content indicates lower elastic modulus and Poisson ratio. Then a higher moisture content of grain, i.e., a lower grain elastic modulus and Poisson ration, asks a lower cylinder speed to decrease impact damage on grain kernel. Obviously, this result is in conflict with that drawn by Eq. (2). The four parameters discussed above should match each other to obtain an optimal combination, improving harvesting productivity and avoiding grain damage.

\section{Optimal parameter matching based on genetic-fuzzy algorithm}

Fuzzy logic control methodology [6] incorporates human and deductive knowledge. It combines four modules to perform three tasks [7]: fuzzification of input, fuzzy inference and defuzzification into output. Genetic algorithm (GA) simulates the natural evolution process to search the global optimal solution.

Fuzzy logic controller of combine harvester. A multiple-input multiple-output fuzzy logic controller is presented, as shown in Fig. 1. Two input variables are feeding rate $(q)$ and grain moisture content $(\beta)$, and two output variables are forward speed $\left(v_{m}\right)$ and cylinder speed $(n)$.

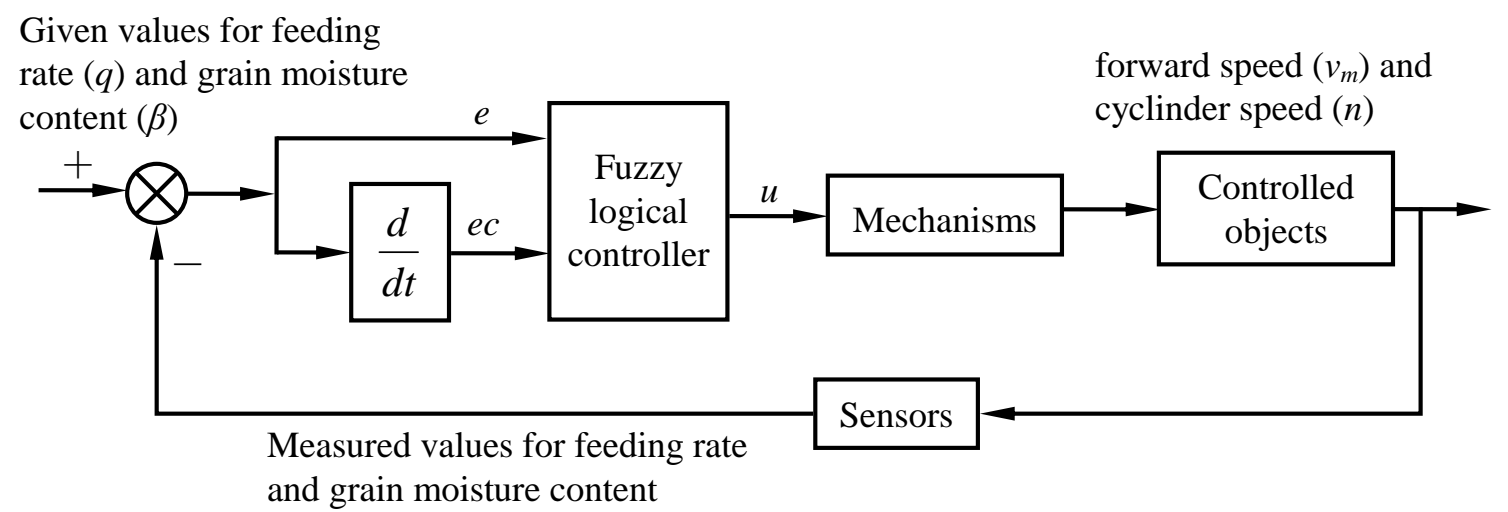

Fig. 1 Fuzzy logic controller of combine harvester 
For the input/output variables mentioned above, basic ranges are $q \in[1,5], \beta \in[15 \%, 30 \%]$, $v_{m} \in[0.2,1.2]$, and $n \in[39,539]$, respectively. All fuzzy ranges (i.e. $q^{\prime}, \beta^{\prime}, v_{m}^{\prime}, n^{\prime}$ ) are from -6 through +6 and are identically defined by seven fuzzy subsets $\{$ NB, NM, NS, O, PS, PM, PB $\}$. Quantitative factors for the input/output variables are defined by $k_{i}, i \in[1,4]$. According to these fuzzy subsets, we can define membership functions (MF), as shown in Fig. 2. For $q^{\prime}$ and $\beta^{\prime}$, we used the same MFs shown in Fig.2 (a), i.e., NB was established by descending semi-trapezoidal MF, PB was determined by ascending semi-trapezoidal MF, and the rest of fuzzy subsets were applied with symmetrically triangular MFs. For $v_{m}^{\prime}$ and $n^{\prime}$, the identical MFs were defined, as shown in Fig. 2(b). Here all fuzzy subsets were determined by making use of symmetrically triangular MFs.

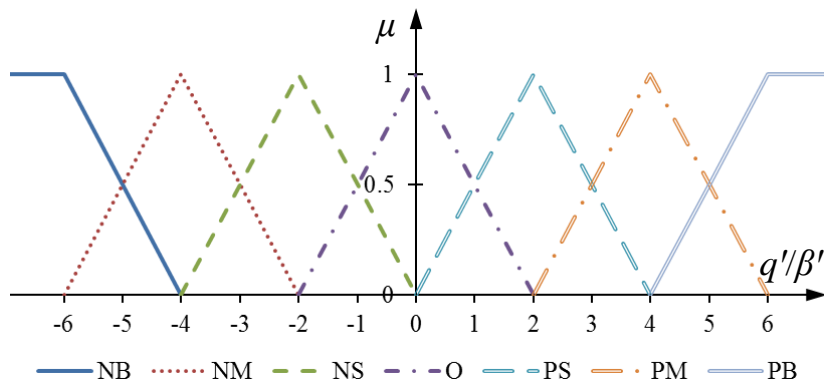

(a)

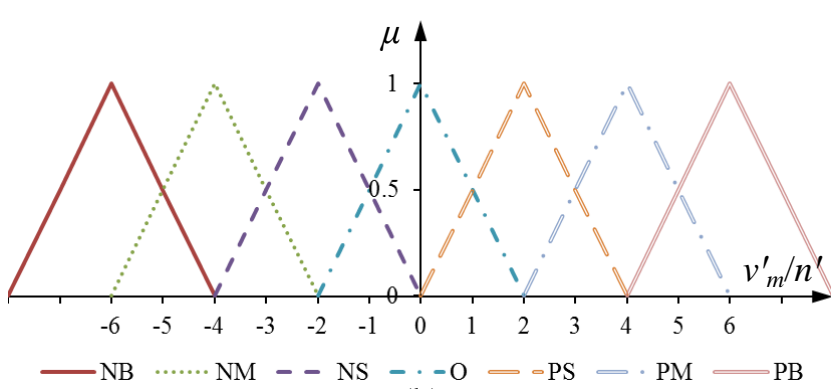

(b)

Fig. 2 Membership function of control variables

Fuzzy rules elucidate relationship between input variables $\left(q^{\prime}\right.$ and $\left.\beta^{\prime}\right)$ and output variables $\left(v_{m}^{\prime}\right.$ and $\left.n^{\prime}\right)$. According to the fuzzy subsets decided above, the fuzzy rules can be defined by

$\mathrm{R}_{i}$ : IF $\left(q_{i}=q_{i}^{\prime}\right)$ and $\left(\beta_{i}=\beta_{i}^{\prime}\right)$ THEN $\left(v_{m i}=v_{m i}^{\prime}\right)$ and $\left(n_{i}=n_{i}^{\prime}\right)$ where $i$ means number of fuzzy rule, namely, $i=7 \times 7=49$.

Fuzzy reasoning and defuzzification are driven by fuzzy rules. We utilize MIN-MAX center of gravity and a singleton fuzzifier to accomplish defuzzification. Fuzzy logic control table of output variables can be defined by fuzzy logical toolbox in MATLAB system. The results were plotted in Fig. 3. Here Fig.3 (a) indicates relationship between output variable $v_{m}^{\prime}$ and input variables $q^{\prime}$ and $\beta^{\prime}$. And Fig. 3 (b) elucidates relationship between output variable $n^{\prime}$ and input variables $q^{\prime}$ and $\beta^{\prime}$.

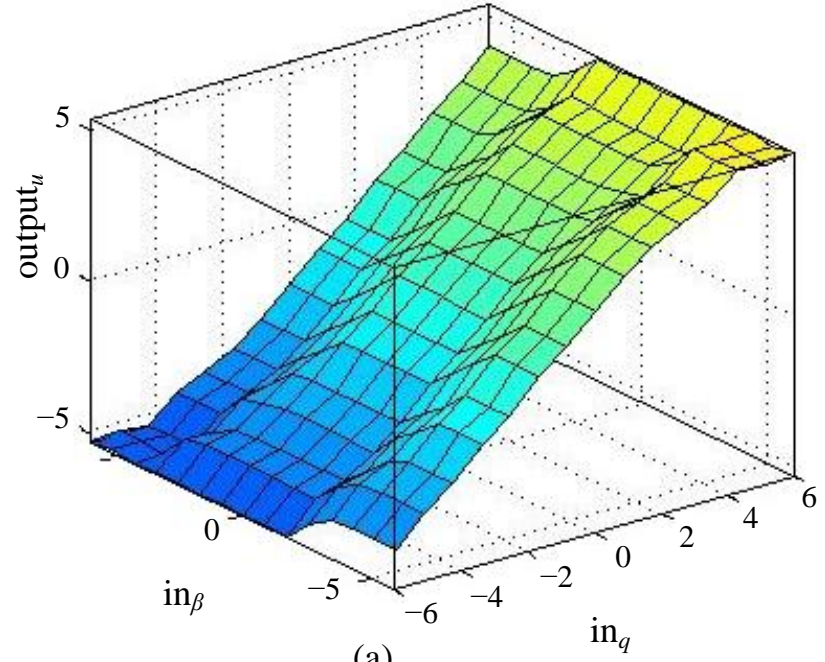

(a)

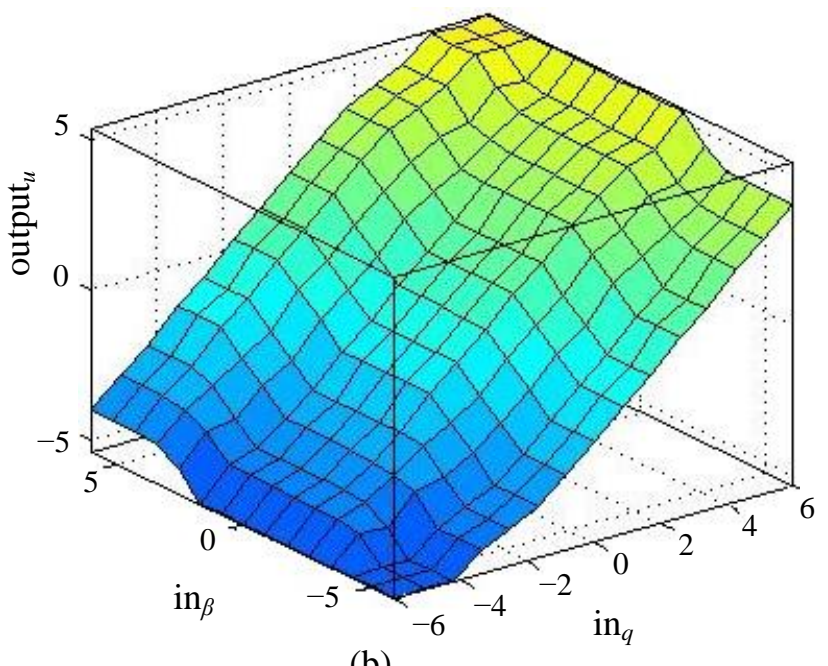

(b)

Fig. 3 Input/output surface of fuzzy logical model

The exact solutions in basic ranges can be obtained in computation of inverse operation with quantitative factors $k$.

Genetic-fuzzy algorithm. The initial individuals are encoded with binary number. For the present fuzzy logic control system, a chromosome asks 1196-bit binary number to encode quantitative factors, MFs and fuzzy rules. Each individual denotes a candidate solution for the optimal combination of input/outpu variables. We take the highest productivity of combine as optimizing object to establish fitness function of GA. Productivity of a combine is given by 


$$
Q_{1}=5.4 \eta B v_{m}
$$

where $\eta, B, v_{m}$ are harvesting time coefficient, cutting width and forward speed, respectively. And productivity of a threshing component is written by

$$
Q_{2}=5.83 n
$$

Using the sum-weighted method, we get the fitness function of optimization as:

Maximize $f=\alpha_{1} \frac{\sum_{i=1}^{n} Q_{1 i}}{n Q_{1 \max }}+\alpha_{2} \frac{\sum_{i=1}^{n} Q_{2 i}}{n Q_{2 \max }}$

with $\alpha_{1}+\alpha_{2}=1$

and where $f$ is evaluation value of fitness function, $n$ is population size, $Q_{1 \max }$ and $Q_{2 \max }$ mean maxima of $Q_{1}$ and $Q_{2}$ in each generation, respectively. And $\alpha_{1}$ and $\alpha_{2}$ are weight coefficients of combine productivity and threshing component productivity. Variables in GA computation are defined as follows. Population size $(n)$ is 40 , and probabilities of crossover and mutation are defined as $p_{c}=0.7$ and $p_{m}=0.05$, respectively. Number of generation is 40 . Finally, we finished computations by using GA toolbox in MATLAB system.

\section{Results and discussion}

Fig. 4 shows results of genetic-fuzzy logic algorithm for parameter matching of a combine. In Fig. 4 (a), the blue curve shows fitness value of the optimal solution (i.e., the maximum of evaluation value), and the red curve denotes average of evaluation value for population. We can observe that average of evaluation value (red curve) rises gradually to a wave curve, indicating a good convergence of the GA computations. At 28th generation on the blue curve, the optimal solution is max fitness value which is 0.81 , corresponding to the optimal parameter matching $q=5, \beta=22.5 \%, v_{m}=1.2$, and $n=539$. Then combine can work at the optimal working condition with the highest productivity.

Each evaluation value of individual in each generation is plotted in Fig. 4 (b). For all individual in each generation, maximum of evaluation value is plotted in a small red circle, and the others are marked with a blue asterisk. Distribute of the red circle for each generation agrees with the curve of optimal solution (see blue curve in Fig. 4 (a)). Spread of the blue asterisks mainly ranges from 0.5 through 0.78 before 10th generation. After 20th generation, most marks locate nearly at the max evaluation value 0.81 and the optimal solution should be the global optimum. Again the fuzzy logic controller is feasible and the genetic-fuzzy algorithm in our work is effective and efficiency.

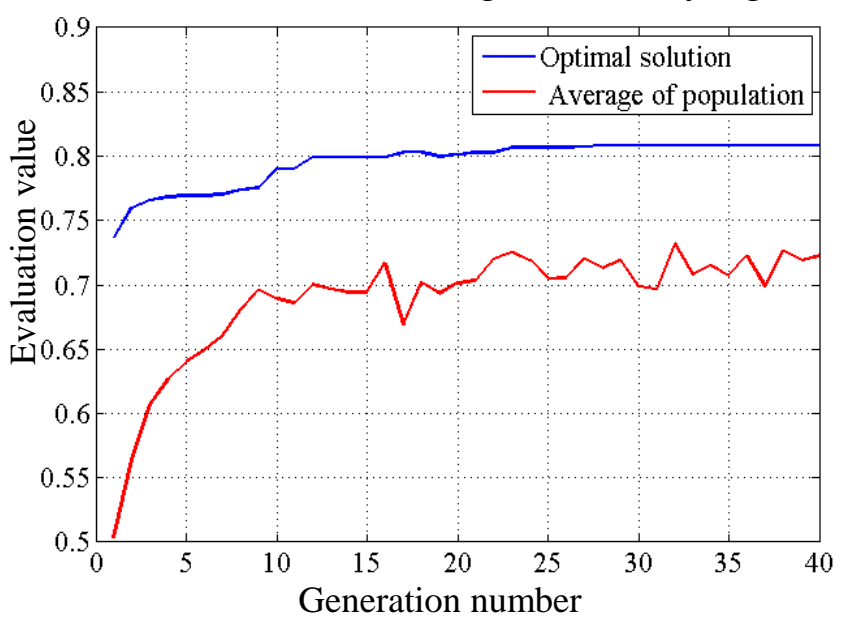

(a)

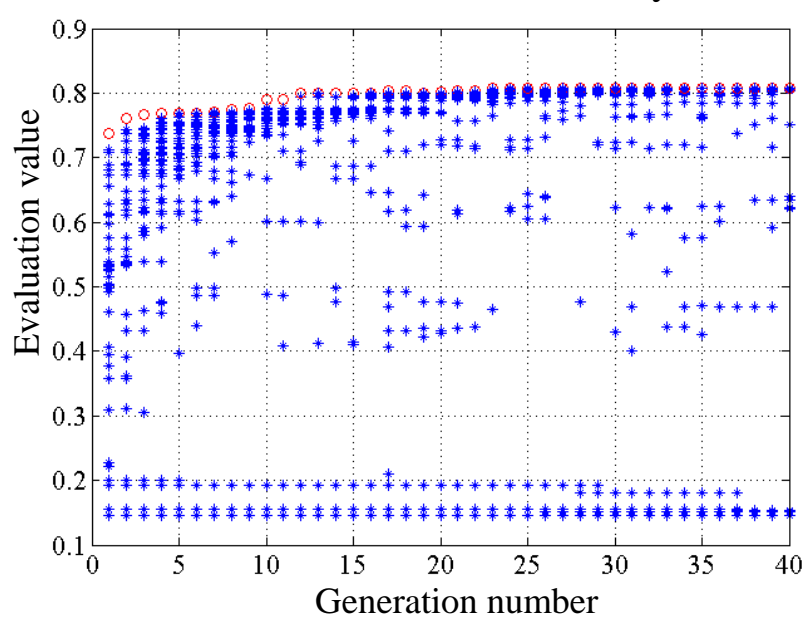

(b)

Fig. 4 Results of genetic-fuzzy logic algorithm 


\section{Summary}

(1) Interrelationship of crucial working parameter of combine harvester has been investigated. A conflict between grain moisture content and cylinder speed was presented, and optimal parameter matching was asked to improve harvesting productivity.

(2) Fuzzy logic controller of combine harvester was proposed by input/output variables of feeding rate, grain moisture content, forward speed and cylinder speed. Genetic-fuzzy logical algorithm was utilized to achieve computations. The results show that the fuzzy logic controller and solving algorithm (i.e. GA) are feasible and efficiency.

\section{Acknowledgements}

Authors are grateful for the supports from the Open Research Subject of Key Lab (Research Base) of Fluid and Power Machinery (No. SZJJ2014-048), Applied Basic Research Program of Sichuan (No. 2014JY0055), Key Technology R\&D Program of Sichuan (No. 2015NZ0055).

\section{References}

[1] W.M. Kotyk, T.G. Kirk, Control system for combine harvesters, Western Canada Conference on Computer, Power and Communications Systems in a Rural Environment, IEEE, 1991, pp. 96-102.

[2] J.B. Zhao, D.A. Zhao C.F. Teng, Intelligent Control System Design of Combine Load Feedback Based on $\mathrm{ARM} 9+\mu \mathrm{C} / \mathrm{OS}$, Second International Conference on Information and Computing Science, IEEE, 2009, pp. 86-89.

[3] M. Omid, Majid Lashgari, Hossein Mobli, Reza Alimardani, Saeid Mohtasebi, Reza Hesamifard, Design of fuzzy logic control system incorporating human expert knowledge for combine harvester, Expert Syst. Appl., 37 (2010) 7080-7085.

[4] C.Y. Tuo, Combined Harvesters' Walking Velocity Analysis and Control System Design, J. of Agr. Mech. Res., 33(2011) 97-100. In Chinese

[5] L.Z Xu, Y.M. Li, Critical Speed of Impact Damage on a Rice Kernel, T. Chinese Soc. Agr. Eng., 40(2007) 54-57. In Chinese

[6] G. Craessaerts, W. Saeys, B. Missotten, J. De Baerdemaeker, Identification of the cleaning process on combine harvesters, Part II: A fuzzy model for prediction of the sieve losses, Biosyst. Eng., 106 (2010) 97-102.

[7] R. Bosma, J. van den Berg, U. Kaymak, H. Udo, J. Verreth, A generic methodology for developing fuzzy decision models, Expert Syst. Appl., 39 (2012) 1200-1210. 